\title{
Genome-wide analysis of proline-rich extension-like receptor protein kinase (PERK) in Brassica rapa and its association with the pollen development
}

Guohu Chen ${ }^{1,2,3^{*}}$ (D), Jian Wang ${ }^{1,2}$, Hao Wang ${ }^{1}$, Chenggang Wang ${ }^{1,2,3^{*}}$, Xiaoyan Tang ${ }^{1,2}$, Jie Lii , Lei Zhang ${ }^{1}$, Jianghua Song ${ }^{1}$, Jinfeng $\mathrm{Hou}^{1}$ and Lingyun Yuan ${ }^{1}$

\begin{abstract}
Background: Proline-rich extension-like receptor protein kinases (PERKs) are an important class of receptor kinases located in the plasma membrane, most of which play a vital role in pollen development.

Results: Our study identified 25 putative PERK genes from the whole Brassica rapa genome (AA). Phylogenetic analysis of PERK protein sequences from 16 Brassicaceae species divided them into four subfamilies. The biophysical properties of the BrPERKs were investigated. Gene duplication and synteny analyses and the calculation of Ka/Ks values suggested that all 80 orthologous/paralogous gene pairs between B. rapa and A. thaliana, B. nigra and B. oleracea have experienced strong purifying selection. RNA-Seq data and QRT-PCR analyses showed that several BrPERK genes were expressed in different tissues, while some BrPERKs exhibited high expression levels only in buds. Furthermore, comparative transcriptome analyses from six male-sterile lines of $B$. rapa indicated that 7 BrPERK genes were downregulated in all six male-sterile lines. Meanwhile, the interaction networks of the BrPERK genes were constructed and 13 PERK coexpressed genes were identified, most of which were downregulated in the male sterile buds.

Conclusion: Combined with interaction networks, coexpression and QRT-PCR analyses, these results demonstrated that two BrPERK genes, Bra001723.1 and Bra037558.1 (the orthologs of AtPERK6 (AT3G18810)), were downregulated beginning in the meiosis II period of male sterile lines and involved in anther development. Overall, this comprehensive analysis of some BrPERK genes elucidated their roles in male sterility.
\end{abstract}

Keywords: Brassica rapa, Comparative genome analysis, Male sterile, Proline-rich receptor-like protein kinase, Transcriptomic analysis

\section{Background}

Unique membrane receptor-like kinases (RKs) have evolved to regulate biological processes in plants $[1,2]$. These plasma membrane RKs perceive various stimuli

\footnotetext{
* Correspondence: cgh@ahau.edu.cn; cgwang@ahau.edu.cn

'Vegetable Genetics and Breeding Laboratory, College of Horticulture, Anhui Agricultural University, Hefei 230036, China

Full list of author information is available at the end of the article
}

and direct them to downstream signaling networks via the phosphorylation of specific domains [3]. In Arabidopsis, approximately 610 RKs have been predicted, which comprise a variety of extracellular ligand-binding domains and are implicated in a wide range of functions, from plant growth and development to plantenvironment interactions $[2,4,5]$. These RK proteins can be grouped into many different classes according to

(c) The Author(s). 2020 Open Access This article is licensed under a Creative Commons Attribution 4.0 International License, which permits use, sharing, adaptation, distribution and reproduction in any medium or format, as long as you give appropriate credit to the original author(s) and the source, provide a link to the Creative Commons licence, and indicate if changes were made. The images or other third party material in this article are included in the article's Creative Commons licence, unless indicated otherwise in a credit line to the material. If material is not included in the article's Creative Commons licence and your intended use is not permitted by statutory regulation or exceeds the permitted use, you will need to obtain permission directly from the copyright holder. To view a copy of this licence, visit http://creativecommons.org/licenses/by/4.0/ The Creative Commons Public Domain Dedication waiver (http://creativecommons.org/publicdomain/zero/1.0/) applies to the data made available in this article, unless otherwise stated in a credit line to the data. 
their extracellular domain motifs [6, 7]. For instance, the leucine-rich repeat receptor kinases (LRR-RKs) are the largest of the RK family in Arabidopsis and sense a diverse set of hormone signaling pathways to regulate cell proliferation and expansion, stomatal development and stem cell maintenance [5, 8-10].

Proline-rich extension-like receptor-kinases (PERKs) are a small family of RKs containing cytoplasmic kinaselike domains, transmembrane motifs and extracellular proline-rich domains $[1,11,12]$. The first PERK gene was identified in Brassica napus, which is rapidly induced by wounding, ubiquitously expressed in the stem, petals and pistils and controls root and stem branching $[13,14]$. Recent work addressing the PERK family of Gossypium hirsutum has revealed the expression patterns of these genes in response to various abiotic stresses and hormonal homeostasis [15]. In addition to these functions, PERKs are thought to act as sensors/receptors to monitor changes in the cell wall during its expansion $[1,12]$.

In Arabidopsis, the PERK family includes 15 members, and the analysis of their expression pattern shows that several of these genes are broadly expressed, while some AtPERK genes are specifically expressed [16]. For example, AtPERK1 (NsAK) is ubiquitously expressed, and AtPERK2 is mainly expressed in rosette leaf veins, stems and pollen [14, 17]; AtPERK4, a $\mathrm{Ca}^{2+}$ signaling regulator, is implicated in root growth and seed germination and is highly expressed in mature pollen and pollen tubes [1820]; AtPERK8 and AtPERK13 (RHS10) are specifically expressed in root hairs [11, 16, 21]; and AtPERK5, AtPERK6, AtPERK7, AtPERK11 and AtPERK12 (IGI1) are highly expressed in pollen but are almost undetectable in sporophytic tissues [1, 11, 22, 23].

Evidence from Arabidopsis shows that most PERKs not only contribute to cell growth and cell wall deposition but also play important roles in male-female interactions and precisely control pollen/pollen tube behaviors [1, 18]. Male gametogenesis requires several metabolic pathways, including the tapetum development, callose metabolism, anther dehiscence, pollen wall and pollen tube wall formation pathways [24-26], and defects in any of these processes can cause abnormal development and ultimately lead to male sterility [26]. However, the molecular mechanisms of PERKs function in pollen development, especially in male sterility, are still unknown.

Thus far, the genome-wide characterization of PERK genes has been reported only in cotton and Arabidopsis $[15,16]$. Little information on the PERK family and their biological functional roles in $B$. rapa are available. Chinese cabbage (B. rapa L.) is one of the most important species in the Brassicaceae family and is widely cultivated in most parts of China [26, 27]. In this study, we performed a systematic analysis of the PERK family in $B$. rapa. The biophysical properties of BrPERK genes were determined. Furthermore, RNA-Seq data from six malesterile plants (two male-sterile mutants [26, 28], two GMS lines [29, 30] and two ogu-CMS lines [27, 31]) were analyzed, and tissue-specific expression patterns were examined to identify the specific PERK genes related to pollen development and male sterility in B. rapa. The present study will contribute to a detailed understanding of the molecular and biological functions of BrPERK genes in male-sterile plants of B. rapa.

\section{Results}

\section{Identification of PERK proteins}

In our study, a total of 418 PERK gene family members in 16 Brassicaceae species were identified based on several confirmations. With the exception of $B$. juncea $(\mathrm{n}=$ 18), B. napus $(\mathrm{n}=19)$ and Camelina sativa $(\mathrm{n}=20)$, the PERK gene numbers in the other 13 species ranged from 17 to 27 (Table S1; Fig. S1), which might be related to their chromosome numbers and indicated that the PERK genes have undergone expansion in Brassicaceae species. A maximum likelihood (ML) tree was generated for these PERK proteins, showing that all PERKs were grouped into four subfamilies (Groups I-IV; Fig. 1, Table S2). Although Group III contained the fewest PERKs (only 22 members), it included PERK members from all Brassicaceae species, and most species exhibit only one PERK (Table S2).

Among Brassica species, 25, 27 and 22 PERK family members were identified in $B$. rapa (AA), B. nigra (BB) and B. oleracea (CC), respectively (Table S1; Fig. S1). The phylogenetic tree of these genes along with those of A. thaliana showed that the total 89 PERKs could again be divided into four groups (Fig. 2a, Groups 1-4). Group 1 and Group 3 constituted the largest clades, containing a total of 65 PERKs, and accounted for 46.07 and $38.20 \%$ of the sequences, respectively. Interestingly, the PERK protein numbers of the three Brassica species in Groups 1-3 were almost the same (Fig. $2 \mathrm{~b}$ ), indicating that these PERK genes from the three Brassica plants may have come from a common ancestor. Furthermore, the sequence logos of the homologous domain sequences of the PERK proteins revealed that the domain sequences were highly conserved in A. thaliana and the three Brassica species (Fig. S2).

We also determined the physical and chemical characteristics of BrPERK proteins. The BrPERK proteins showed wide variation in their length, molecular weight (MW) and isoelectric point (pI) (Table S3). Moreover, the majority of the proteins were localized to the chloroplast thylakoid membrane, followed by the endoplasmic reticulum (membrane), plasma membrane, cytoplasm, nucleus and chloroplast stroma (Table S3). 


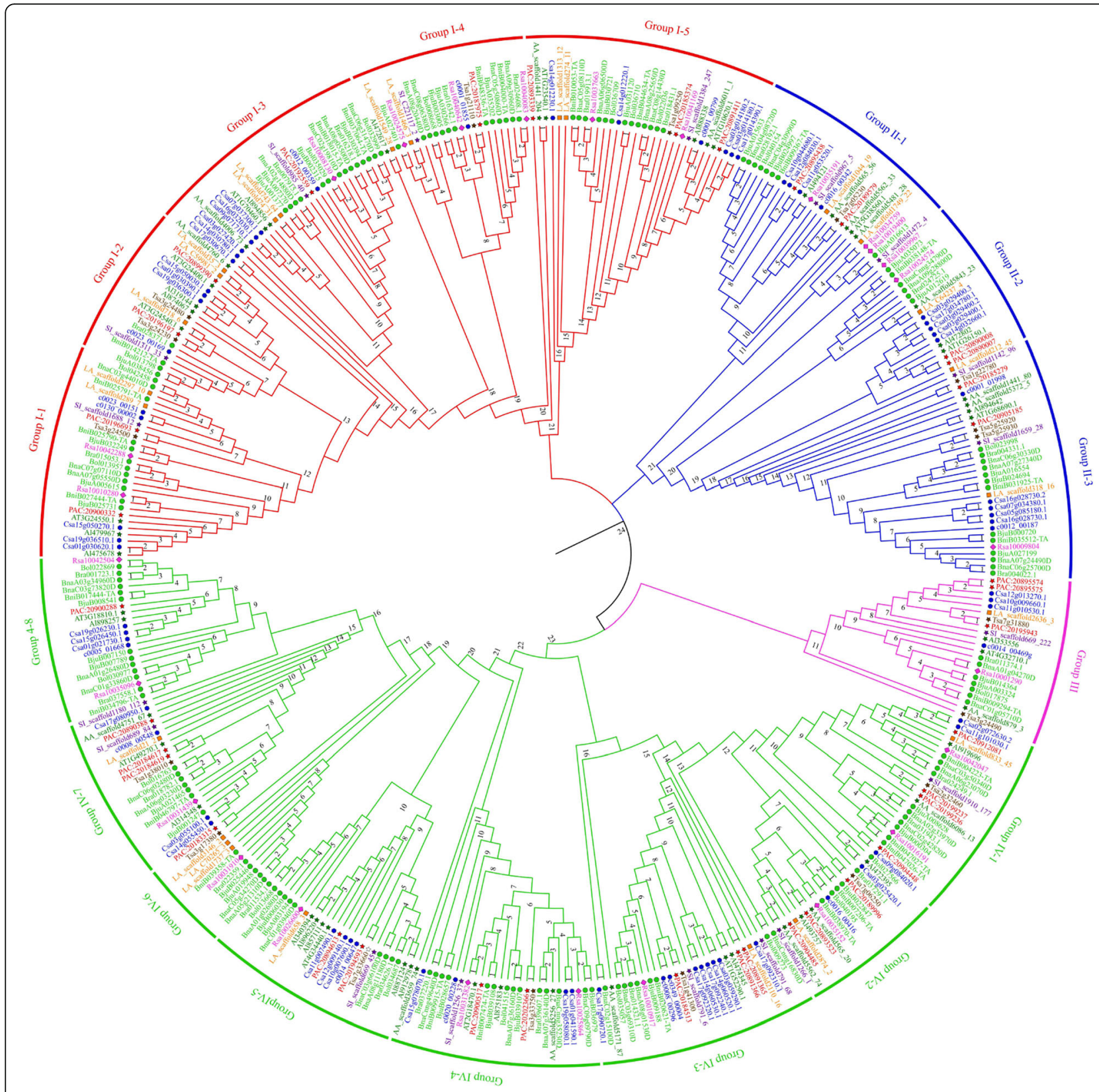

Fig. 1 Phylogenetic analysis of PERK proteins from 16 Brassicaceae species. 418 PERK proteins are grouped into four subfamilies. The gene number of 16 Brassicaceae species in different groups was calculated in Table S2. Percent bootstrap values (1000 iterations) are indicated in every branch. The different-colored arcs indicate different groups of PERKs. The maximum likelihood (ML) tree was constructed by MEGA7 with the Bootstrap method (1000 replications) and the Jones, Taylor, and Thornton amino acid substitution (JTT) model

\section{Conserved motifs, gene structures and cis-elements analyses}

The functional motifs and gene exon-intron positions were analyzed based on evolutionary tree relationships (Fig. 3). The ten most conserved motifs were identified in the BrPERK members (Fig. S3), showing that almost all of the BrPERK proteins contained the same motifs in terms of both the type and distribution patterns of the motifs, except for Bra037558 (Fig. 3b). The exon-intron organization suggested that all BrPERK genes showed conserved patterns of gene structure, and all conserved motifs were associated with the Pkinase domain (Pkc like superfamily and STKc_IRAK) (Fig. 3c). Overall, the highly similar gene structures and motif distributions of the BrPERK members were consistent with their phylogenetic relationships (Fig. 3). cis-element analysis showed that several promoters contained meristem expression motifs, and a few promoters contained 

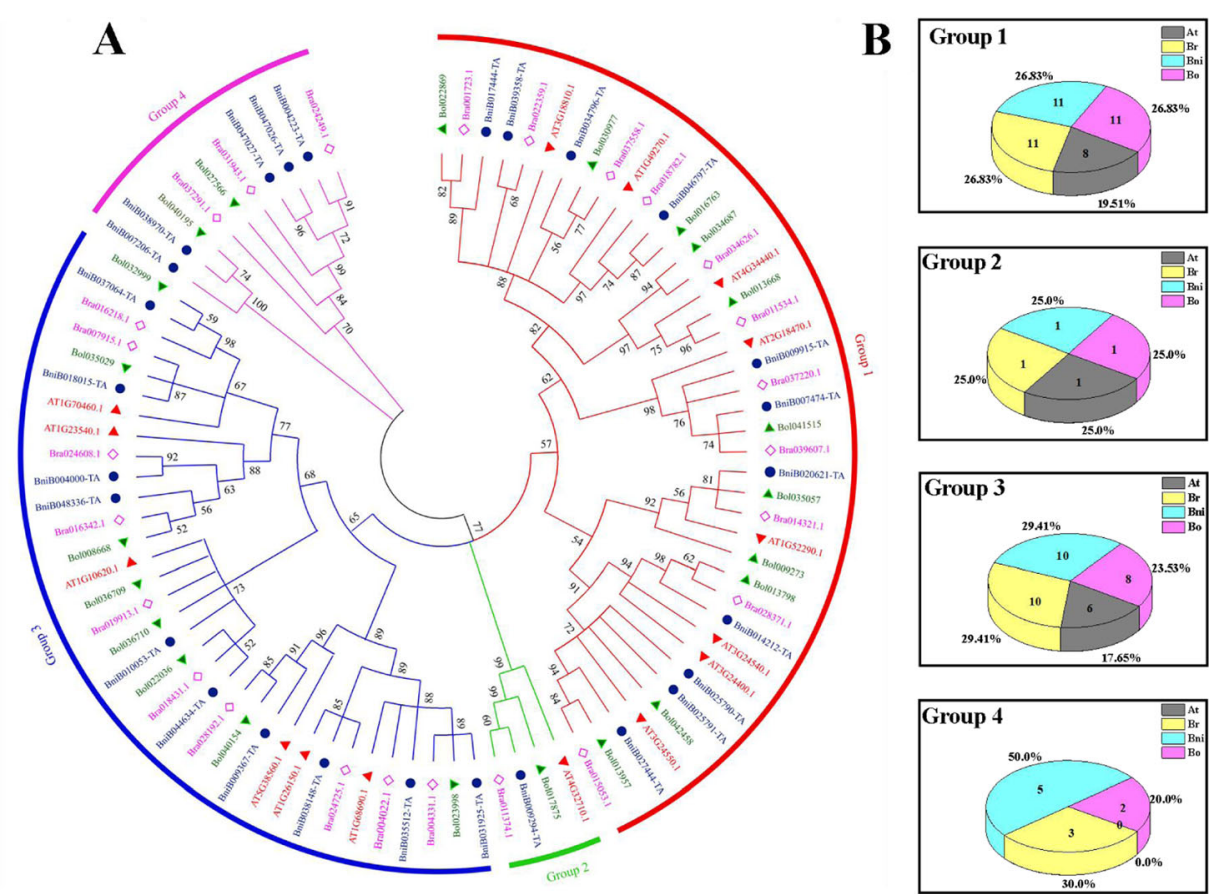

Fig. 2 Phylogenetic analysis and distribution of PERK proteins from four plant species. a, Phylogenetic tree of $B$. rapa, B. nigra, B. oleracea, and $A$. thaliana. 89 PERKs from them are divided into four groups. The different-colored arcs indicate different groups of PERKs. The maximum likelihood (ML) tree was constructed by MEGA7 with the Bootstrap method (1000 replications) and the Jones, Taylor, and Thornton amino acid substitution (JTT) model. b, Number and percentage of PERK proteins across the four plant species within each subfamily. At, Arabidopsis thaliana; Br, Brassica rapa; Bni, Brassica nigra; Bo, Brassica oleracea

endosperm and seed-specific expression, cell cycle regulation and circadian control elements (Table S4; Fig. S4), indicating the roles of several PERK genes in plant flowering.

\section{Gene duplication, synteny and $K a / K s$ analyses}

The mapping of the BrPERK genes to chromosomal loci showed that an inconsistent distribution of the genes, with 1 to 5 genes being distributed on chromosomes A1-A9 (Fig. S5A; Table S3). Synteny and $\mathrm{Ka} / \mathrm{Ks}$ analyses indicated that 13 paralog pairs were distributed on different chromosomes, not including the A04 and A10 chromosomes (Table S5, Fig. S5B). The $K s$ values ranged from 0.29 to 1.23 , and the average duplication time of paralog pairs was indicated to be 22.84 million years ago (Mya) (Table S5, Fig. S6A), possibly because some BrPERK gene duplications occurred before the whole-genome triplication observed in B. rapa.

Further analyses of PERK gene evolution and divergence among A. thaliana and the three Brassica species showed that a total of 67 orthologous gene pairs exhibited a collinear relationship (17 Br-At, $27 \mathrm{Br}$ -
Bni, 23 Br-Bo; Fig. 4, Table S5). These results demonstrated that the PERK genes of these Brassica species appeared to be derived from a common ancestor and that the function of these PERK genes of Brassica plants might be the same as those of $A$. thaliana. In addition, among the orthologous gene pairs, each AtPERK gene presented 1-3 BrPERK orthologous genes (Fig. 4 and S7, Table S5), suggesting that a few BrPERK genes had been duplicated by genome triplication. The $\mathrm{Ka} / \mathrm{Ks}$ values of these gene pairs were all less than 0.35 except for one pair (Bra028371.1-Bo1013798, Ka/Ks = 0.56), and the average divergence times were estimated to be 17.12 Mya (Br-At), 11.96 Mya (Br-Bni), and 8.95 Mya (Br-Bo) (Table S5, Fig. S6B-D). These results demonstrated that the PERK gene pairs shared between $B$. rapa and $B$. nigra, B. oleracea, and A. thaliana had undergone strong purifying selection with limited functional divergence after whole-genome duplication (13-17 Mya [32]).

\section{Tissue-specific expression pattern analysis}

The RNA-Seq data from different tissues of $B$. rapa showed that the BrPERK members exhibited variable 


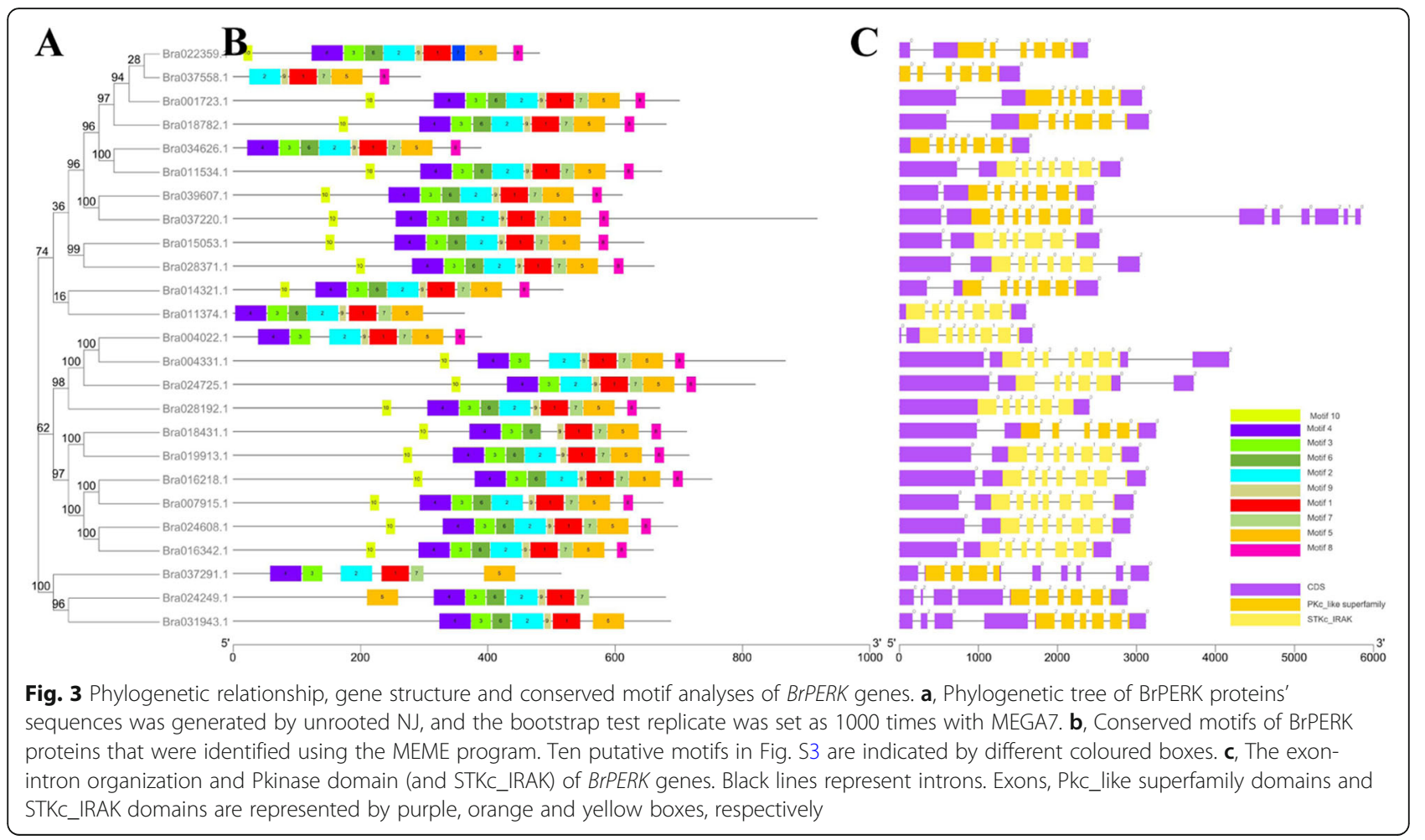

expression patterns; several genes were broadly expressed in all different tissues, while some members were exhibited a more tissue-specific pattern and were especially highly expressed in flower tissue (Fig. 5a). The qRT-PCR results from different organs confirmed the RNA-Seq data, and several BrPERK genes were highly expressed in sepals and petals (Fig. $5 \mathrm{~b})$. Furthermore, BrPERK gene expression differed in different anther developmental stages, with some of these genes exhibiting high expression in the uninucleate, binucleate and mature pollen periods (Fig. 5c-d and S8). Together, these results suggested that some BrPERK genes may play important roles in flower development, especially in pollen development.

Comparative transcriptome analysis of BrPERK genes in $B$. rapa male-sterile lines

Transcriptome datasets from six different male-sterile lines were analyzed, including two ogura cytoplasmic male-sterile (ogu-CMS) lines (wucai and turnip), two genic male-sterile (GMS) lines (AB01 and AB03) and two male-sterile mutants ( $m s m$ and ftms). A total of 14 differentially expressed genes (DEGs) of BrPERKs were detected, and seven of them were downregulated in all male-sterile (MS) plants (Fig. 6 and S9A). qRT-PCR analysis of different developing buds showed that these BrPERK genes presented variable expression patterns, and most of them were downregulated after the tetrad period (Fig. $6 \mathrm{~b}$ and c). These results demonstrated that these downregulated BrPERK genes participated in anther development.

\section{Regulatory subnetworks involving BrPERKs and other genes}

To gain further insight into the roles of these downregulated BrPERK genes in anther development, interaction networks of the BrPERK genes in Chinese cabbage were constructed. From the STRING database, 19 coexpressed genes were identified (Fig. 7a). RNA-Seq data from 6 male-sterile lines and qRT-PCR results were analyzed to assess the expression patterns of these genes. Ten genes were identified in at least 2 male lines (Fig. 7b), and most of the genes were downregulated in these male sterile buds (Fig. 7b). The interaction network of these genes with BrPERKs and their expression levels during pollen development are shown in Fig. 7c and d, respectively. Gene Ontology (GO) analysis indicated that 8 genes were enriched in pectinesterase activity, nucleic acid binding, cation binding, the membrane, and protein kinase activity; Kyoto Encyclopedia of Genes and Genomes (KEGG) analysis showed that three genes were involved in starch and sucrose metabolism, metabolic pathways, and pentose and glucuronate interconversion (Table S6). 


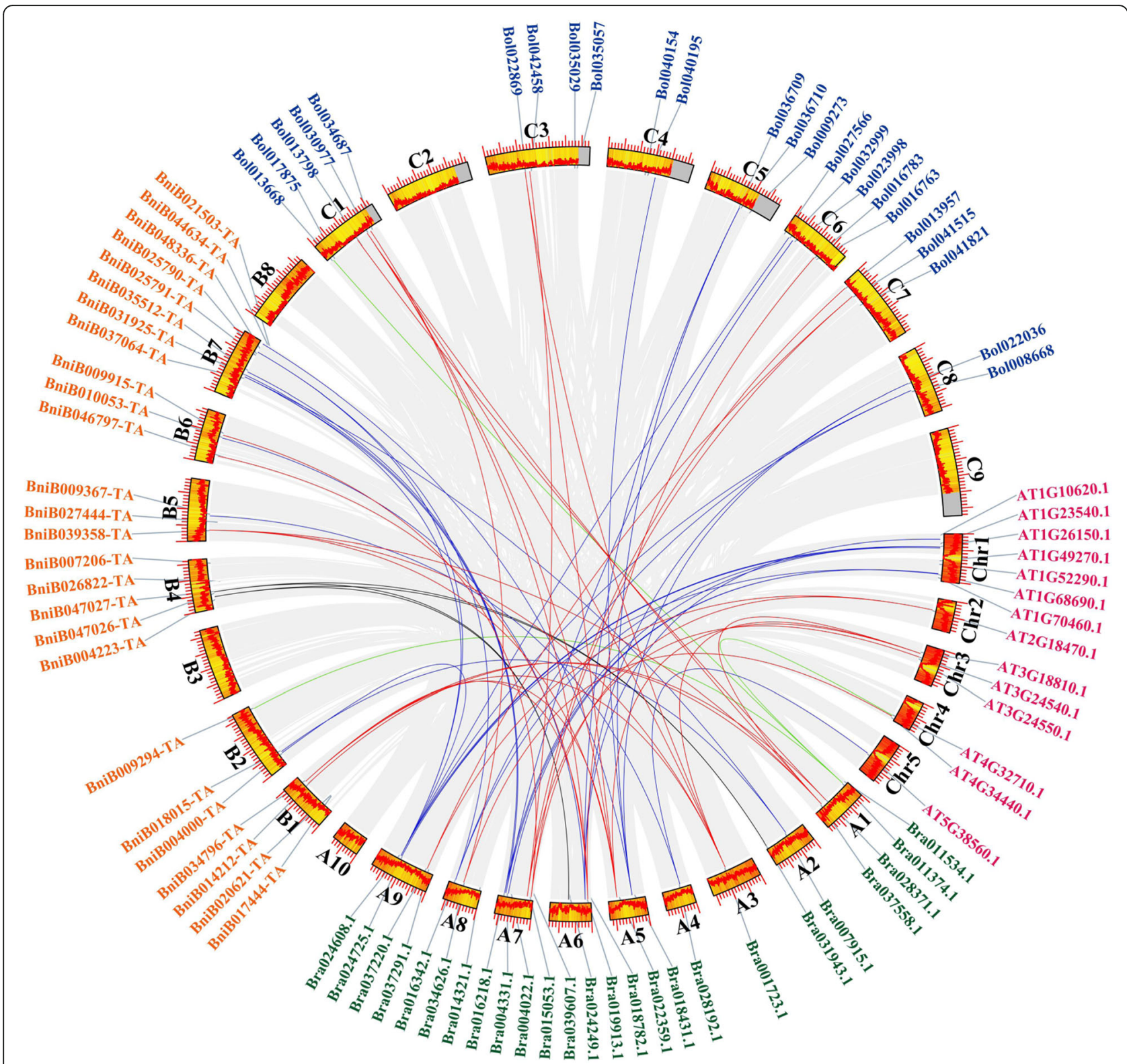

Fig. 4 PERK gene duplication among B. rapa (AA) and A. thaliana, B. nigra (BB), B. oleracea (CC). Different color lines exhibited paralogous pairs. Chr1-Chr5 (red blocks) indicated chromosomes of A. thaliana; A1-A10 (green blocks), B1-B8 (orange blocks) and C1-C9 (blue blocks) represented sub-genomes of $B$. rapa (AA), B. nigra (BB) and B. oleracea (CC), respectively

\section{Discussion}

In the present study, we successfully identified 25 BrPERK members from the whole-genome sequence of $B$. rapa and analyzed their biophysical properties (Table S3). However, 6 more BrPERK members were identified in our analyses that have been reported in previous studies [1]. Among the 25 BrPERKs, 6 members (Bra022359, Bra037558, Bra034626, Bra011374, Bra004022 and Bra037291) were shorter, which generally lacked partial sequences in the $5^{\prime}$ flanking region (Fig. 3c); all of these proteins are extracellular proteins (Table S3) and exhibit similar patterns of the main motif distribution (Fig. 3b). These shortened proteins might exist because partial sequences were deleted in the process of gene duplication [33, 34]. Nevertheless, these 6 genes still belong to the PERK gene family, and they were retained in the subsequent analyses. Unlike AtPERK members $[1,13,16]$, all of the proline-rich regions of BrPERKs contained less than 2 Ser-Pro ${ }_{(2-3)}$ motifs in the $5^{\prime}$ flanking region (data not shown).

In addition to $B$. rapa and $A$. thaliana, we identified PERKs in 14 other Brassicaceae species (Table S1), and the number of PERKs did not differ greatly in these 


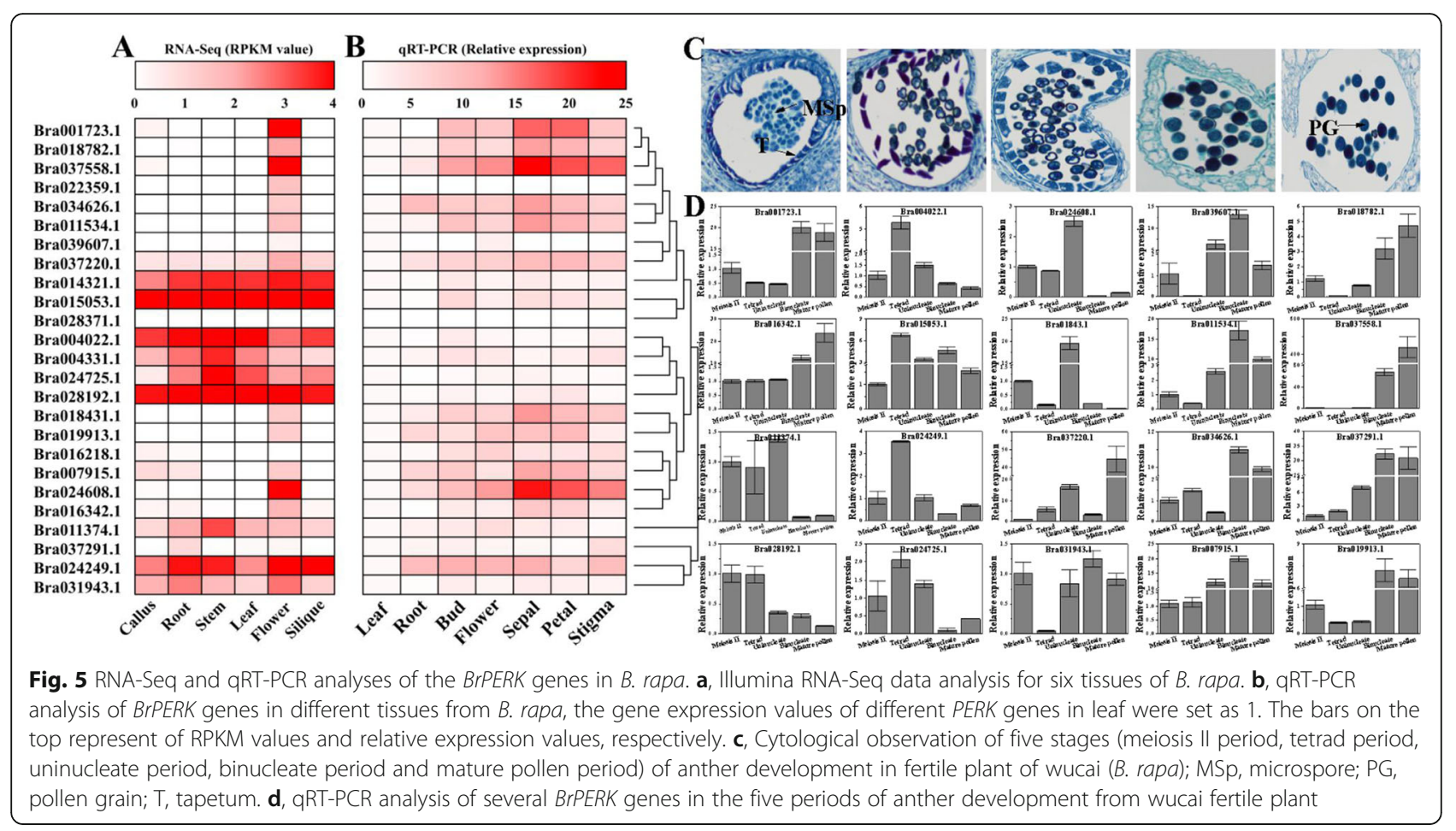

species except in three polyploid plant species, suggesting that the PERK family is well conserved in these species [17]. The phylogenetic tree of a total of 418 PERKs showed that Group III among the four subgroups is an ancient clade, similar to the findings from a previous phylogenetic tree of 207 PERKs from 15 other plant species [15]. Interestingly, in Group III, most of the Brassicaceae species presented only one PERK member, except for $C$. rubella $(\mathrm{n}=8)$ and $T$. salsuginea $(\mathrm{n}=7)$ and three polyploid plant species, B. juncea $(\mathrm{n}=18), B$. napus $(\mathrm{n}=$ 19) and $C$. sativa $(\mathrm{n}=20)$ (Fig. 1 , Table $\mathrm{S} 2)$, which exhibited far more PERK members than other diploid plant species (Fig. S1, Table S1). These results revealed that the PERK gene family is affected by polyploidy and has experienced segmental and genome duplication in these species $[15,35]$.

The phylogenetic tree of the three Brassica species with A. thaliana showed that Groups 1-3 included almost equal numbers of PERKs in these three Brassica species, while Group 4 lacked an AtPERK gene (Fig. 2); the PERK genes of this group might acquire new functions via divergence following duplication [33, 34, 36, 37]. Furthermore, the sequence logos of the conserved PERK amino acid residues showed that the PERK genes are evolutionarily conserved among the Brassica species and A. thaliana (Fig. S2). This situation can be attributed to the fact that $B$. rapa underwent whole-genome duplication twice in the Brassicaceae lineage and experienced an additional wholegenome triplication event [32, 34].
Additionally, we estimated the divergence times of the orthologous PERK pairs between $B$. rapa and A. thaliana, $B$. nigra, and B. oleracea (Fig. 4 and S7), which showed $\mathrm{Ka} / \mathrm{Ks}$ ratios less than 0.35 and indicating the occurrence of strong purifying selection (Fig. S6, Table S5), reflecting the strong control exerted over these genes in evolution [38]. Moreover, the average divergence times of $B$. rapa from $A$. thaliana, B. nigra, and B. oleracea were estimated to be 17.12, 11.96 and 8.95 Mya, respectively (Table S5, Fig. S6), indicating that Brassica species shared a common ancestor and exhibit different differentiation times [32, 35, $39,40]$. It has been reported that the divergence time of the genomes of $B$. rapa and A. thaliana was approximately 13 to 17 Mya [41]. These results imply that the PERK gene family might be a good candidate molecular reference for analysis of plant evolution. In addition, we analyzed BrPERK gene duplication events within the $B$. rapa genome, which are thought to play important roles in the amplification of the genome [42, 43]. In B. rapa, 13 paralog pairs of duplicated BrPERK genes have been retained, but none of the gene pairs exhibit tandem duplication (Fig. S5A), suggesting that segmental duplication events have played a leading role in BrPERK family evolution, similar to what is observed in the IQD gene family of B. rapa [44].

It has been reported that AtPERK members are involved in several biochemical pathways related to developmental processes in A. thaliana, such as pathways that are active in root, rosette leaf, stem and pollen 


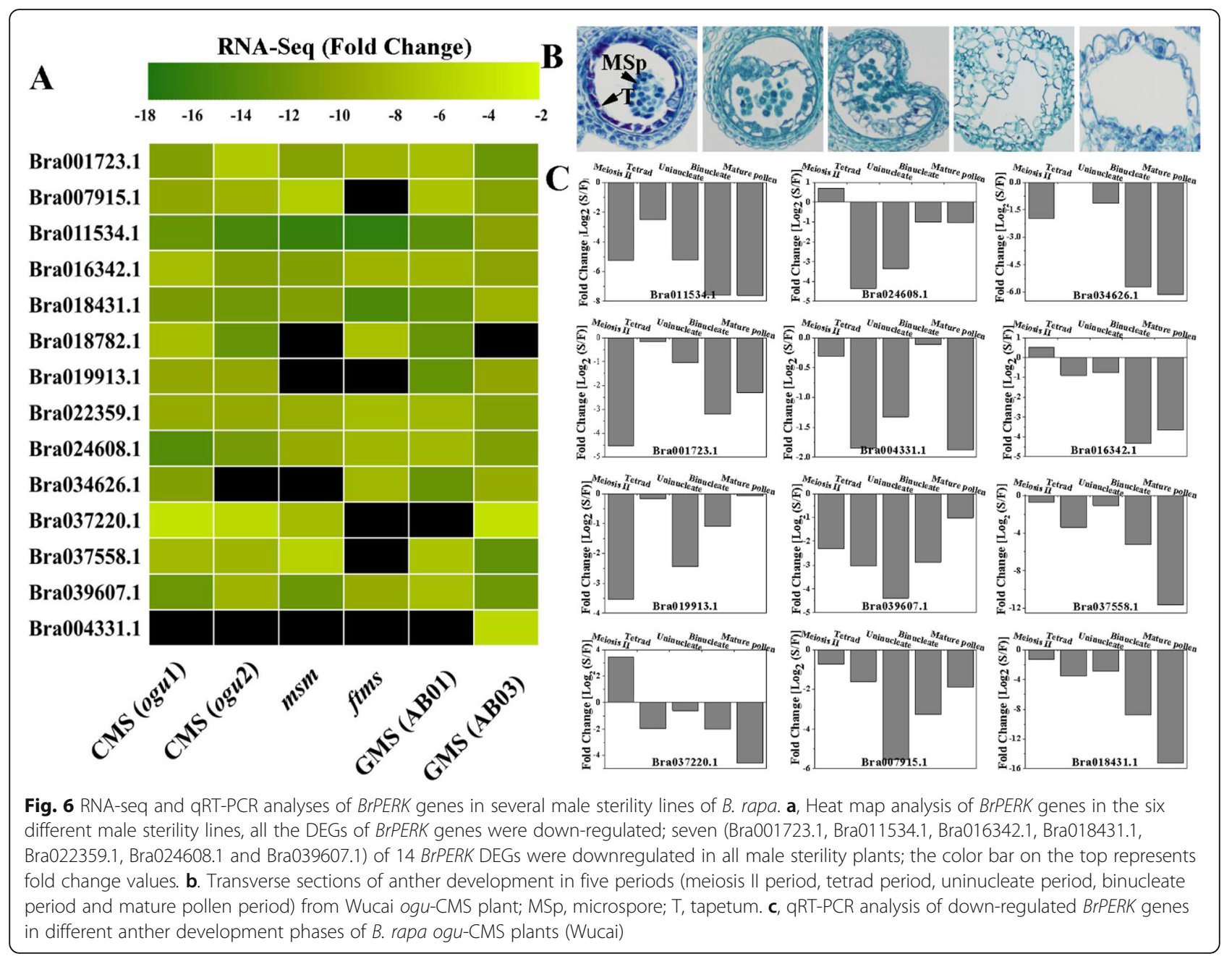

tissues $[1,11,16]$. In B. rapa, the functions of BrPERKs were explored on the basis of RNA-Seq datasets and qRT-PCR results (Fig. 5). Among different tissues, the gene expression levels of different BrPERK genes clearly differed, and some of these genes were very highly and specifically expressed in reproductive organs. These results were similar to those obtained in A. thaliana [11, 16] and Gossypium hirsutum [15], suggesting that some PERK genes play important roles in reproductive developmental processes and might be related to sterility and fertility.

Thus, we assayed 6 RNA-Seq datasets from $B$. rapa male-sterile mutants [26, 28], GMS [29, 30] and CMS $[27,31]$ lines, and found that 9 BrPERK genes were downregulated in at least five MS lines (Fig. 6a). Among these genes, Bra024608.1 and Bra016342.1 were paralogous genes and they were all orthologs of IGI1 (AT1G23540); Bra022359.1, Bra001723.1 and Bra037558.1 were also paralogous genes, and they were all orthologs of AtPERK6 (Fig. 4, Table S5). IGI1 is strongly expressed in the anthers, and the igil mutant exhibited semisterility and smaller siliques [22]. In addition, a phosphoproteomic study of mature Arabidopsis pollen grains showed that AtPERK6 (AT3G18810) was phosphorylated during pollen grain development [23]. In our study, Bra024608.1 and Bra016342.1 were found to be downregulated during anther development from the tetrad period onward, and Bra001723.1 and Bra037558.1 were downregulated beginning in the meiosis II period (Bra022359.1 could not be detected; Fig. $6 \mathrm{~b}$ and $\mathrm{c}$ ). Thus, these results suggested that the four BrPERK genes might be related to male sterility.

Further analysis of the coexpression network revealed 19 genes that were coexpressed with 13 downregulated BrPERKs (except Bra004331.1 in Fig. 6a) screened by STRING (Fig. 7a), and 13 coexpressed genes were identified in the 6 male-sterile RNA-Seq datasets, almost all of which were also downregulated (Fig. 7b). Among these coexpressed genes, no gene coexpressed with Bra024608.1 and Bra016342.1 was screened; nevertheless, 7 genes coexpressed with Bra001723.1 and Bra037558.1 were identified (Fig. 7c, Table S6). In addition, it was reported that 


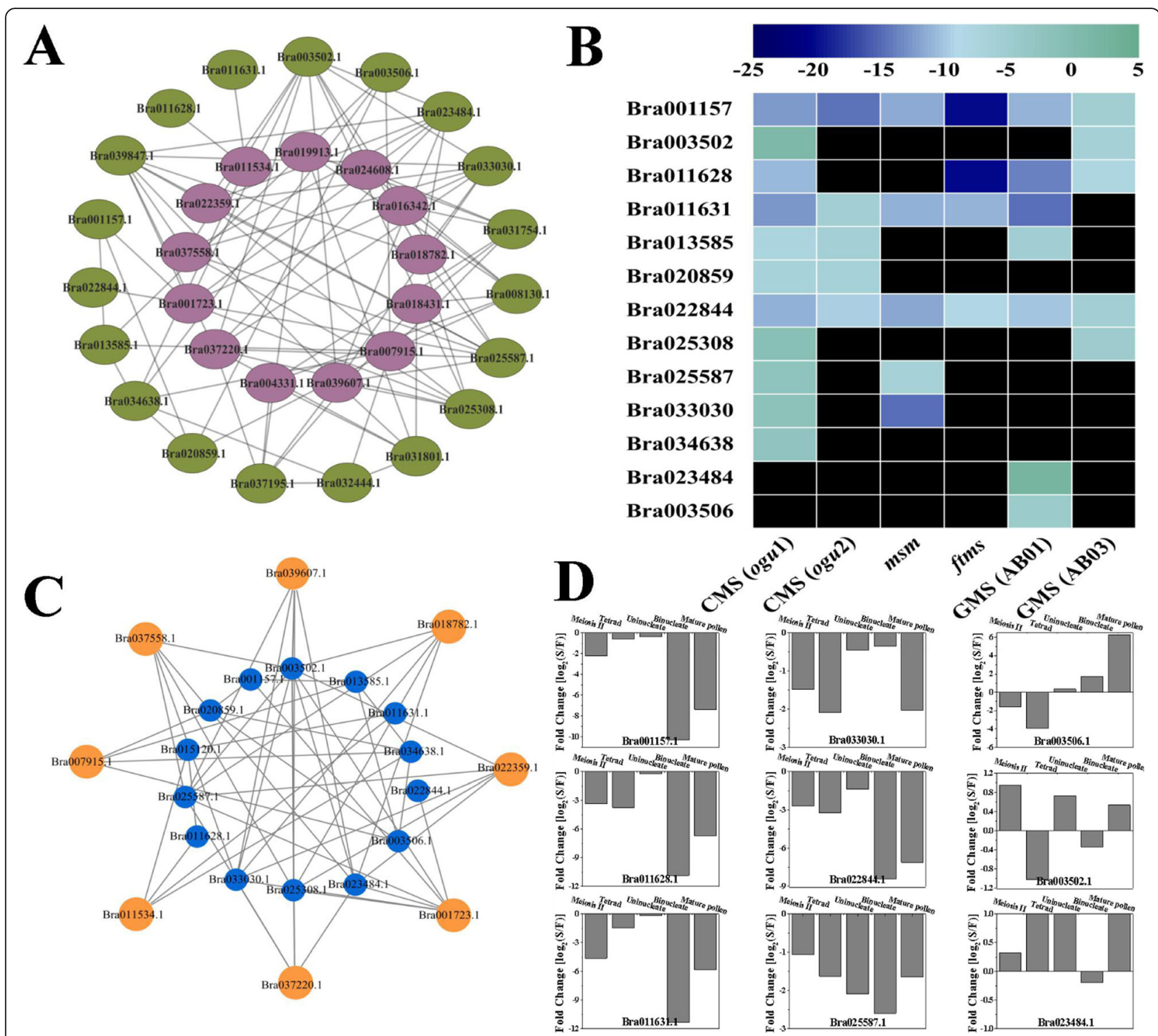

Fig. 7 Co-expression, RNA-seq data and qRT-PCR analyses of BrPERK co-expressed genes. a. Co-expression network of BrPERK co-expressed genes was visualized; Nodes are connected by lines to show the interaction of that gene to other co-expressed gene; Reddish brown color indicates 13 down-regulated BrPERK genes and green color represent their co-expressed genes. $\mathbf{b}$, Heat map analysis of BrPERK co-expressed genes in the six different male sterility lines; the color bar on the top represents fold change values. c. Co-expression network of BrPERK co-expressed genes; Orange color indicates 8 down-regulated BrPERK genes and blue color represent their co-expressed genes. $\mathbf{d}$, qRT-PCR analysis of down-regulated BrPERK co-expressed genes in B. rapa ogu-CMS plants (Wucai)

Bra001723.1 (Unigen37636) was participated in anther and pollen development [27], and this gene was involved in starch and sucrose metabolism (ko00500) and other metabolic pathways (ko01100) (Table S6). These results demonstrated that Bra001723.1 and Bra037558.1 interact in the regulation of anther and pollen development and the downregulation of these genes might be related to male sterility in B. rapa. However, further research is required to investigate the detailed regulatory mechanisms of Bra001723.1 and Bra037558.1 related to male sterility in $B$. rapa.

\section{Conclusions}

In this study, we identified 418 PERK gene family members in 16 Brassicaceae species and characterized 25 BrPERK genes in B. rapa. Analyses of phylogenetic relationships, conserved motifs, gene structures, cis-elements, gene duplication and synteny were performed to comprehensively evaluate their biophysical properties in B. rapa. Additionally, expression pattern assays based on RNA-Seq and qRT-PCR data revealed that some PERKs present tissue-specific patterns and are involved in reproductive development, especially pollen development. 
Combined analyses of RNA-Seq datasets from six malesterile lines of $B$. rapa demonstrated that 13 BrPERK genes were downregulated in the buds of at least four male-sterile lines, possibly indicating crucial roles in pollen tissue. The expression profiles of these BrPERK genes showed that Bra024608.1, Bra016342.1, Bra001723.1 and Bra037558.1 were downregulated during anther development, suggesting that their abnormal expression might be related to male sterility. Furthermore, the coexpression networks assay exhibited that only Bra001723.1 and Bra037558.1 can be detected the coexpressed genes among these four PERK genes. These results provided basic information for studying the mechanism of Bra001723.1 and Bra037558.1 function in the male sterility of $B$. rapa.

\section{Methods}

\section{Identification of PERK genes in 16 Brassicaceae species}

The sequences of AtPERK family proteins were downloaded from the TAIR website following Nakhamchik et al. [16] and served as queries in BLAST searches to identify PERK members in the other 15 Brassicaceae species in the Brassica Database (BRAD) [32]. After the removal of redundant sequences, all candidate protein sequences were verified using the SwissProt database [45] and the SMART [46], Pfam [47] and InterProScan tools [48]. The confirmed protein sequences were aligned and used to generate a phylogenetic tree in MEGA 7.0 [49]. The conserved amino acid sequence logos were constructed with the WEBLOG tool [50].

\section{Structural analysis of PERKs}

The biophysical properties, protein localization sites, signal peptides, and transmembrane helices of the proteins were predicted using the ExPASy program [51], ProtComp (v6), PSORT Prediction, SignalP [52] and TMHMM Servers (v2.0), respectively. Information on the exon/intron structure, conserved motifs and domains of BrPERKs was obtained from BRAD with the MEME [53] and CDD [54] tools. The predicted motifs were annotated by InterProScan [48]. Tbtools (v0.6679) [55] was used for visual analysis. The promoter regions (1.5 kb upstream sequences) of all BrPERK genes were used to predict cis-acting elements in the PlantCARE database [56], and promoter structures were obtained with the GSDS (v2.0) [57] tool.

\section{Genomic distribution and collinearity analyses}

The distribution of the BrPERK genes on chromosomes was searched and plotted using Tbtools. Synteny analysis among the three Brassica crops and A. thaliana genomes was conducted via all against all BLASTP comparisons. The entire protein sequences were used as queries to search the corresponding protein sequence data $\left(E<1 \mathrm{e}^{-5}\right.$, top 5 matches). Collinear pairs were extracted using MCScanX [58] to identify syntenic blocks and duplications within the PERKs.

\section{Calculation of $K_{a} / K_{s}$ values}

The rates of synonymous $\left(K_{s}\right)$ and nonsynonymous $\left(K_{a}\right)$ substitutions were calculated for duplicated BrPERK genes with the KaKs calculator [59]. $K_{s}$ values $>2.0$ were discarded to avoid the risk of substitution saturation, and the divergence time was calculated according to Khan et al. [35] and Yuan et al. [44].

\section{RNA-Seq data analysis}

Seven raw RNA-Seq datasets of B. rapa plant were obtained from the NCBI Gene Expression Omnibus (GEO) and Sequence Read Archive (SRA) [26-31, 60]. One of these datasets consisted of the genome-wide transcriptomes of tissues [60], and the other six datasets consisted of the transcriptomes of male-sterile lines, including two male-sterile mutants $[26,28]$, two GMS lines $[29,30]$ and two ogu-CMS lines [27, 31]. These male-sterile lines are no pollen phenotype and their anther abortion occurs during the tetrad period. The raw data were filtered and mapped onto the B. rapa genomes (V1.5 and V3.0). Gene expression levels were estimated using Reads Per Kilobase of exon model per Million mapped reads (FPKM) values [61], and differentially expressed genes (DEGs) were determined with the DESeq or DEGseq software package [62]. The transcriptome expression of PERKs was illustrated via heatmap analysis [27].

\section{Microscopy observations, gene expression and interaction network analyses}

The ogu-CMS line 4-2B and its 4-2A maintainer line from wucai (B. rapa ssp. Chinensis var. rosularis Tsen) were used as the plant materials in this study, as described previously [63, 64]. Different developmental stages of buds from CMS and fertile lines were used for paraffin section and qRT-PCR analyses (Table S7) according to our previous reports $[27,63,65]$. The interaction network associated with BrPERK genes was constructed using the STRING database [66] and Cytoscape software [67].

\section{Supplementary information}

Supplementary information accompanies this paper at https://doi.org/10. 1186/s12864-020-06802-9.

Additional file 1: Table S1. Gene locus ID of 16 Brassicaceae plants from Brassica Database.

Additional file 2: Table S2. Statistics of PERKs in different groups of phylogenetic tree from 16 Brassicaceae species.

Additional file 3: Table S3. Biophysical properties of BrPERK genes. 
Additional file 4: Table S4. Characterization of the promoter ciselement prediction of BrPERK genes.

Additional file 5: Table S5. Orthologous / paralogous in B. rapa and A. thaliana, B. nigra and B. oleracea.

Additional file 6: Table S6. GO and KEGG enrichment analyses of BrPERKS Co-expressed genes.

Additional file 7: Table S7. List of all primers used in this study. Additional file 8: Figure S1. Number and percentage of PERK proteins across the 16 Brassicaceae species.

Additional file 9: Figure S2. Sequence logos of conserved amino acid residues in Arabidopsis thaliana and three Brassica species (B. rapa, B. nigra and B. oleracea).

Additional file 10: Figure S3. Sequence logos of conserved amino acid residues in BrPERKs.

Additional file 11: Figure S4. Cis-acting regulatory element analysis of BrPERKs promoters by PlantCARE.

Additional file 12: Figure S5. Chromosomal location (A) and gene duplication (B) of BrPERK genes on their corresponding chromosomes.

Additional file 13: Figure S6. Ka/Ks values and divergence times of orthologous / paralogous gene pairs. A, Br-Br; B, Br-At; C, Br-Bni; D, Br-Bo.

Additional file 14: Figure S7. BrPERK gene duplication analysis between B. rapa (AA) and A. thaliana, B. nigro (BB) and B. oleracea (CC).

Additional file 15: Figure S8. qRT-PCR analysis of three BrPERK genes in the five periods of anther development.

Additional file 16: Figure: S9. Venn plots of BrPERK DEGs (A) and BrPERK co-expression genes (B) in six B. rapa male sterile lines.

\section{Abbreviations}

BRAD: Brassica Database; CMS: Cytoplasmic male sterile; DEG: Differentially expressed gene; FPKM: Reads per kilobase of exon model per million mapped reads; GEO: Gene Expression Omnibus; GMS: Genetic male sterile; GO: Gene ontology; KEGG: Kyoto encyclopedia of genes and genomes; LRRRK: Leucine-rich repeat receptor kinase; ML: Maximum likelihood; MW: Molecular weight; MS: Male-sterile; Mya: Million years ago; PERK: Prolinerich extension-like receptor protein kinase; pl: Isoelectric point; qRTPCR: Quantitative real-time PCR; RK: Receptor-like kinase; RNA-Seq: RNA sequencing; SRA: Sequence read archive

\section{Acknowledgements}

We are grateful to the Researcher Feng Cheng, Dr. Kang Zhang and Ms. Yingqing Yang (Institute of Vegetables and Flowers, CAAS) for providing the advances and platform for bioinformatic analysis.

\section{Authors' contributions}

GC designed the research. GC and JW carried out the experiments, and GC wrote the manuscript. HW and XT helped to analyze the data. JL and LZ helped to perform the experiments. CW, JS, JH and LY helped to draft the manuscript. All authors have read and approved the final manuscript.

\section{Funding}

National Natural Science Foundation of China (31801853); Outstanding Talents Cultivation Project in Colleges and Universities (gxgnfx2019001); Anhui Provincial Natural Science Foundation (1908085MC96); Key Projects of Natural Science Research Funds in Support of Colleges (KJ2017A153).

\section{Availability of data and materials}

All data generated or analyzed during this study are included in this published article [and its supplementary information files].

\section{Ethics approval and consent to participate}

Not applicable.

\section{Consent for publication}

Not applicable.

\section{Competing interests}

The authors declare that they have no competing interests.

\section{Author details}

${ }^{1}$ Vegetable Genetics and Breeding Laboratory, College of Horticulture, Anhui Agricultural University, Hefei 230036, China. ${ }^{2}$ Anhui Provincial Engineering Laboratory of Horticultural Crop Breeding, Hefei 230036, China. ${ }^{3}$ Wanjiang Vegetable Industrial Technology Institute, Maanshan 238200, China.

Received: 7 March 2020 Accepted: 2 June 2020

Published online: 15 June 2020

\section{References}

1. Borassi C, Sede AR, Mecchia MA, Salgado Salter JD, Marzol E, Muschietti JP, Estevez JM. An update on cell surface proteins containing extensin-motifs. J Exp Bot. 2016;67(2):477-87

2. Hohmann U, Lau K, Hothorn M. The structural basis of ligand perception and signal activation by receptor kinases. Annu Rev Plant Biol. 2017;68(1): 109-37.

3. Osakabe Y, Yamaguchishinozaki K, Shinozaki K, Tran LSP. Sensing the environment: key roles of membrane-localized kinases in plant perception and response to abiotic stress. J Exp Bot. 2013:64(2):445-58.

4. Muschietti JP, Wengier DL. How many receptor-like kinases are required to operate a pollen tube. Curr Opin Plant Biol. 2018;41:73-82.

5. Gish LA, Clark SE. The RLK/Pelle family of kinases. Plant J. 2011;66(1):117-27.

6. Shiu SH, Bleecker AB. Plant receptor-like kinase gene family: diversity, function, and signaling. Science's Stke. 2001;113:re22.

7. Shin Han S, Bleecker AB. Expansion of the receptor-like kinase/Pelle gene family and receptor-like proteins in Arabidopsis. Plant Physiol. 2003;132(2): 530-43.

8. Smakowska-Luzan E, Mott GA, Parys K, Stegmann M, Howton TC, Layeghifard M, Neuhold J, Lehner A, Kong J, Grunwald K, et al. An extracellular network of Arabidopsis leucine-rich repeat receptor kinases. Nature. 2018;553(7688):342-6.

9. Brandt B, Hothorn M. SERK co-receptor kinases. Curr Biol. 2016:26(6):225-6.

10. Peng X, Wang M, Li Y, Yan W, Chang Z, Chen Z, Xu C, Yang C, Wang Deng $X$, Wu J, et al. Lectin receptor kinase OsLecRK-S.7 is required for pollen development and male fertility. J Integr Plant Biol. 2019. https://doi.org/10. 1111/jipb.12897.

11. Humphrey TV, Haasen KE, Aldeabrydges MG, Sun H, Zayed $Y$, Indriolo $E$, Goring DR. PERK-KIPK-KCBP signalling negatively regulates root growth in Arabidopsis thaliana. J Exp Bot. 2015;66(1):71-83.

12. Humphrey TV, Bonetta DT, Goring DR. Sentinels at the wall: cell wall receptors and sensors. New Phytol. 2007:176(1):7-21.

13. Silva NF, Goring DR. The proline-rich, extensin-like receptor kinase-1 (PERK1) gene is rapidly induced by wounding. Plant Mol Biol. 2002:50(4):667-85.

14. Haffani YZ, Silva-Gagliardi NF, Sewter SK, Aldea MG, Zhao Z, Nakhamchik A, Cameron RK, Goring DR. Altered expression of PERK receptor kinases in Arabidopsis leads to changes in growth and floral organ formation. Plant Signal Behav. 2006;1(5):251-60.

15. Qanmber G, Liu J, Yu D, Liu Z, Lu L, Mo H, Ma S, Wang Z, Yang Z. Genomewide identification and characterization of the PERK gene family in Gossypium hirsutum reveals gene duplication and functional divergence. Int J Mol Sci. 2019;20:1750.

16. Nakhamchik A, Zhao Z, Provart NJ, Shiu S-H, Keatley SK, Cameron RK, Goring DR. A comprehensive expression analysis of the Arabidopsis Proline-rich Extensin-like receptor kinase gene family using bioinformatic and experimental approaches. Plant Cell Physiol. 2004;45(12):1875-81.

17. Florentino LH, Santos AA, Fontenelle MR, Pinheiro GL, Zerbini FM, BaracatPereira MC, Fontes EP. A PERK-like receptor kinase interacts with the geminivirus nuclear shuttle protein and potentiates viral infection. J Virol. 2006;80(13):6648-56.

18. Ling B, Guozeng Z, Yun Z, Zhaopei Z, Wei W, Yanyan D, Zhongyi W, ChunPeng S. Plasma membrane-associated proline-rich extensin-like receptor kinase 4, a novel regulator of $\mathrm{Ca}^{2+}$ signalling, is required for abscisic acid responses in Arabidopsis thaliana. Plant J. 2009:60(11):314-27.

19. Bai $L$, Zhou Y, Song C. Arabidopsis proline-rich extensin-like receptor kinase 4 modulates the early event toward abscisic acid response in root tip growth. Plant Signal Behav. 2009;4(11):1075-7.

20. Qin Y, Leydon Alexander R, Manziello A, Pandey R, Mount D, Denic S, Vasic $B$, Johnso MA, Ravishanka P. Penetration of the stigma and style elicits a 
novel transcriptome in pollen tubes, pointing to genes critical for growth in a pistil. PLoS Genet. 2009;5(8):e1000621.

21. Won S, Lee Y, Lee H, Heo Y, Cho M, Cho H. Cis-element- and transcriptomebased screening of root hair-specific genes and their functional characterization in Arabidopsis. Plant Physiol. 2009;150(3):1459-73.

22. Indeok H, Soo Young K, Cheol Soo K, Yoonkyung P, Giri Raj T, Seong-Ki K, Hyeonsook C. Over-expression of the IG/1 leading to altered shootbranching development related to MAX pathway in Arabidopsis. Plant Mol Biol. 2010;73(6):629-41.

23. Mayank P, Grossman J, Wuest S, Boisson-Dernier A, Roschitzki B, Nanni P, Nühse T, Grossniklaus U. Characterization of the phosphoproteome of mature Arabidopsis pollen. Plant J. 2012;72(1):89-101.

24. Chen L, Liu YG. Male sterility and fertility restoration in crops. Annu Rev Plant Biol. 2014;65(1):579-606.

25. Wu Y, Min L, Wu Z, Yang L, Zhu L, Yang X, Yuan D, Guo X, Zhang X. Defective pollen wall contributes to male sterility in the male sterile line 1355A of cotton. Sci Rep. 2015;5:9608.

26. Tan C, Liu Z, Huang S, Li C, Ren J, Tang X, Liu W, Peng S, Feng H. Pectin methylesterase inhibitor (PMEI) family can be related to male sterility in Chinese cabbage (Brassica rapa ssp. pekinensis). Mol Gen Genomics. 2018; 293(2):343-57.0000.

27. Chen G, Ye X, Zhang S, Yuan L, Zhu S, Hou J, Wang C. Comparative transcriptome analysis between fertile and CMS flower buds in Wucai (Brassica campestris L.). BMC Genomics. 2018;19:908.

28. Huang S, Peng S, Liu Z, Li C, Tan C, Yao R, Li D, Li X, Hou L, Feng H. Investigation of the genes associated with a male sterility mutant $(\mathrm{msm})$ in Chinese cabbage (Brassica campestris ssp. pekinensis) using RNA-Seq. Mol Genet Genomics. 2019. https://doi.org/10.1007/s00438019-01618-z.

29. Liu C, Liu Z, Li C, Zhang Y, Feng H. Comparative transcriptome analysis of fertile and sterile buds from a genetically male sterile line of Chinese cabbage. In Vitro Cell Dev Biol Plant. 2016;52(2):130-9.

30. Zhou X, Liu Z, Ji R, Feng H. Comparative transcript profiling of fertile and sterile flower buds from multiple-allele-inherited male sterility in Chinese cabbage (Brassica campestris L. ssp. pekinensis). Mol Gen Genomics. 2017; 292:967-90.

31. Lin S, Miao Y, Su S, Xu J, Jin L, Sun D, Peng R, Huang L, Cao J. Comprehensive analysis of Ogura cytoplasmic male sterility-related genes in turnip (Brassica rapa ssp. rapifera) using RNA sequencing analysis and bioinformatics. Plos One. 2019;14(6):e0218029.

32. Wang XW, Wang HZ, Wang J, Sun RF, Wu J, Liu SY, Bai YQ, Mun JH, Bancroft I, Cheng F, et al. The genome of the mesopolyploid crop species Brassica rapa. Nat Genet. 2011:43(10):1035-U1157.

33. Khan N, Hu C-M, Khan WA, Wang W, Ke H, Dong H, Zhang Z, Hou X. Genome-wide identification, classification, and expression pattern of homeobox gene family in Brassica rapa under various stresses. Sci Rep. 2018;8:16265

34. Cheng F, Mandáková T, Wu J, Xie Q, Lysak MA, Wang X. Deciphering the diploid ancestral genome of the mesohexaploid Brassica rapa. Plant Cell. 2013;25:1541-54.

35. Khan N, Ke H, Hu C-M, Naseri E, Haider MS, Ayaz A, Khan WA, Wang J, Hou $X$. Genome-wide identification, evolution, and transcriptional profiling of PP2C gene family in Brassica rapa. Biomed Res Int. 2019:2965035.

36. Conant $\mathrm{G}$, Wolfe $K$. Turning a hobby into a job: how duplicated genes find new functions. Nat Rev Genet. 2008;9(12):938-51.

37. Yang Z, Qian G, Qin W, Yang Z, Yuan C, Lu L, Ge X, Zhang C, Wu Z, Li F. Genome-wide analysis of WOX genes in upland cotton and their expression pattern under different stresses. BMC Plant Biol. 2017;17:113.

38. Liu T, Yu H, Xiong X, Yue X, Yu Y, Huang L, Cao J. Genome-wide identification, molecular evolution, and expression profiling analysis of pectin methylesterase inhibitor genes in Brassica campestris ssp. chinensis. Int J Mol Sci. 2018;19(5):1338.

39. Beilstein MA, Nagalingum NS, Clements MD, Manchester SR, Sarah M. Dated molecular phylogenies indicate a Miocene origin for Arabidopsis thaliana. PNAS. 2010;107(43):18724-8.

40. Yang YW, Lai KN, Tai PY, Li WH. Rates of nucleotide substitution in angiosperm mitochondrial DNA sequences and dates of divergence between Brassica and other Angiosperm lineages. J Mol Evol. 1999;48(5): 597-604.

41. Town CD, Cheung F, Maiti R, Crabtree J, Haas BJ, Wortman JR, Hine EE, Althoff R, Arbogast TS, Tallon LJ, et al. Comparative genomics of Brassica oleracea and Arabidopsis thaliana reveal gene loss, fragmentation, and dispersal after polyploidy. Plant Cell. 2006;18(6):1348-59.

42. Zhao T, Wang J, Zhang B, Hou X. Genome-wide analysis of lectin receptor-like kinases in tomato (Solanum lycopersicum) and its association with the infection of tomato yellow leaf curl virus. Plant Mol Biol Rep. 2018;36(3):429-38.

43. Mühlhausen S, Kollmar M. Whole genome duplication events in plant evolution reconstructed and predicted using myosin motor proteins. BMC Evol Biol. 2013;13(1):202.

44. Yuan J, Liu T, Yu Z, Li Y, Ren H, Hou X, Li Y. Genome-wide analysis of the Chinese cabbage IQD gene family and the response of BrIQD5 in drought resistance. Plant Mol Biol. 2019;99(6):603-20.

45. Bairoch A, Apweiler R. The SWISS-PROT protein sequence database and its supplement TrEMBL in 2000. Nucleic Acids Res. 2000;28(1):45-8.

46. Letunic I, Doerks T, Bork P. SMART: recent updates, new developments and status in 2015. Nucleic Acids Res. 2015;43:257-60.

47. Finn RD, Coggill P, Eberhardt RY, Eddy SR, Mistry J, Mitchell AL, Potter SC, Punta M, Qureshi M, Sangradorvegas A. The Pfam protein families database: towards a more sustainable future. Nucleic Acids Res. 2016:44:279-85.

48. Jones P, Binns D, Chang H, Fraser M, Li W, McAnulla C, McWilliam H, Maslen J, Mitchell A, Nuka G, et al. InterProScan 5: genome-scale protein function classification. Bioinf. 2014;30(9):1236-40.

49. Kumar S, Stecher G, Tamura K. MEGA7: molecular evolutionary genetics analysis version 7.0 for bigger datasets. Mol Bio Evol. 2016;33(7):1870-4.

50. Crooks GE, Hon G, Chandonia JM, Brenner SE. WebLogo: A sequence logo generator. Genome Res. 2019;14:1188-90.

51. Gasteiger E, Hoogland C, Gattiker A, Duvaud S, Wilkins MR, Appel RD, Bairoch A. Protein Identification and Analysis Tools on the ExPASyServer. In: Walker J.M. (eds) The Proteomics Protocols Handbook. Springer Protocols Handbooks. 2005: Humana Press.

52. Armenteros JJA, Tsirigos KD, Sønderby CK, Petersen TN. Ole Winther SB, Heijne Gv, Nielsen H. SignalP 5.0 improves signal peptide predictions using deep neural networks. Nat Biotechnol. 2019;37:420-3.

53. Bailey TL, Nadya W, Chris M, Li WW. MEME: discovering and analyzing DNA and protein sequence motifs. Nucleic Acids Res. 2006;34(Web Server issue): 369-73.

54. Marchlerbauer A, Bo Y, Han L, He J, Lanczycki CJ, Lu S, Chitsaz F, Derbyshire MK, Geer RC, Gonzales NR. CDD/SPARCLE: functional classification of proteins via subfamily domain architectures. Nucleic Acids Res. 2017:45:200-3.

55. Chen C, Xia R, Chen H, He Y. TBtools, a Toolkit for Biologists integrating various HTS-data handling tools with a user-friendly interface. bioRxiv. 2018. https://doi.org/10.1101/289660.

56. Magali L, Patrice D, Gert T, Kathleen M, Yves M, Yves VDP, Pierre R, Stephane R. PlantCARE, a database of plant cis-acting regulatory elements and a portal to tools for in silico analysis of promoter sequences. Nucleic Acids Res. 2002;30(1):325-7.

57. Hu B, Jin J, Guo AY, Zhang H, Luo J, Gao G. GSDS 2.0: an upgraded gene feature visualization server. Bioinf. 2014;31 (8):1296-7.

58. Wang $Y$, Tang H, Debarry JD, Tan X, Li J, Wang X, Lee T, Jin H, Marler BS, Guo H. MCScanX: a toolkit for detection and evolutionary analysis of gene synteny and collinearity. Nucleic Acids Res. 2012;40(7):e49.

59. Wang D, Zhang Y, Zhang Z, Zhu J, Yu J. KaKs_Calculator 2.0: a toolkit incorporating gamma-series methods and sliding window strategies. Genomics, Proteom Bioinf. 2010:8(1):79-82.

60. Tong C, Wang X, Yu J, Jian W, Li W, Huang J, Dong C, Wei H, Liu S. Comprehensive analysis of RNA-seq data reveals the complexity of the transcriptome in Brassica rapa. BMC Genomics. 2013;14(1):1-10.

61. Trapnell C, Williams B, Pertea G, Mortazavi A, Kwan G, van Baren M, Salzberg S, Wold B, Pachter L. Transcript assembly and quantification by RNA-Seq reveals unannotated transcripts and isoform switching during cell differentiation. Nat Biotechnol. 2010;28(5):511-5.

62. Anders $\mathrm{S}$, Huber W. Differential expression analysis for sequence count data. Genome Biol. 2010;11(10):R106.

63. Zhang S, Wang J, Chen G, Ye X, Zhang L, Zhu S, Yuan L, Hou J, Wang C. Functional analysis of a MYB transcription factor BrTDF1 in the tapetum development of Wucai (Brassica rapa ssp.). Sci Horic. 2019;257: 108728

64. Chen G, Zeng F, Wang J, Ye X, Zhu S, Yuan L, Hou J, Wang C. Transgenic Wucai (Brassica campestris L.) produced via Agrobacterium-mediated anther transformation in planta. Plant Cell Rep. 2019;38(5):577-86. 
65. Chen G, Ye X, Zeng F, Wang J, Yuan L, Zhu S, Hou J, Cheng Y, Wang C. Characterization and utilization of a cytoplasmic male sterility line of Wucai (Brassica campestris L.). Hortic Environ Biotech. 2019;60(3):373-82.

66. Szklarczyk D, Gable AL, Lyon D, Junge A, Wyder S, Huerta-Cepas J, Simonovic M, Doncheva NT, Morris JH, Bork P, et al. STRING v1 1: protein-protein association networks with increased coverage, supporting functional discovery in genome-wide experimental datasets. Nucleic Acids Res. 2018;47(D1):607-13.

67. Shannon P, Markiel A, Ozier O, Baliga NS, Wang JT, Ramage D, Amin N, Schwikowski B, Ideker T. Cytoscape: a software environment for integrated models of biomolecular interaction networks. Genome Res. 2003;13(11): 2498-504.

\section{Publisher's Note}

Springer Nature remains neutral with regard to jurisdictional claims in published maps and institutional affiliations.

Ready to submit your research? Choose BMC and benefit from:

- fast, convenient online submission

- thorough peer review by experienced researchers in your field

- rapid publication on acceptance

- support for research data, including large and complex data types

- gold Open Access which fosters wider collaboration and increased citations

- maximum visibility for your research: over $100 \mathrm{M}$ website views per year

At $\mathrm{BMC}$, research is always in progress.

Learn more biomedcentral.com/submissions 